\title{
Synthesis and properties of alkylthioethanals
}

\author{
Natalia A. Keiko,* Ludmila G. Stepanova, Ekaterina A. Verochkina, \\ and Yurii A. Chuvashev
}

\author{
A.E. Favorsky Irkutsk Institute of Chemistry, Siberian Branch of the Russian Academy of \\ Sciences, 664033 Irkutsk, Russian Federation \\ E-mail:keiko@irioch.irk.ru
}

\begin{abstract}
A method, allowing alkylthioethanals to be synthesized in $80-93 \%$ yields, has been developed. It comprises the hydrolysis of bis-1,1-alkoxy-2-alkylthioethanes using two-phase system water organic solvent. The method is applicable for both common aldehydes bearing lower radicals ( $\mathrm{R}$ $=\mathrm{Pr}, \mathrm{Bu})$ and hitherto unknown highly boiling aldehydes $\left(\mathrm{R}=\mathrm{C}_{7} \mathrm{H}_{15}, \mathrm{C}_{8} \mathrm{H}_{17}, \mathrm{PhCH}_{2}\right)$. It has been found that alkylthioethanals can spontaneously trimerize into 2,4,6-alkylthiotrioxanes. In basic media $\left(1 \mathrm{~N} \mathrm{NaOH}, \mathrm{KF} / \mathrm{Si}(\mathrm{OEt})_{4} / \mathrm{EtOH}\right)$, alkylthioethanals affords the products of aldol selfcondensation in up to $60 \%$ yield $\left({ }^{1} \mathrm{H}\right.$ NMR). In competitive interaction (cross-aldol condensation) of butylthio- and alkoxyethanals with furan- or thiophen-2-carbaldehydes, the reactivity of sulfur-containing carbanions was shown to be more than six times higher than that of the alkoxy analogs.
\end{abstract}

Keywords: Alkylthioethanal, 1,3,5-trioxanes, aldol self-condensation, cross-aldol condensation, furan- and thiophen-2-carbaldehydes, $\mathrm{CH}$-anion from alkylthioethanals

\section{Introduction}

Alkylthioethanals occur widely in volatile compounds, which contribute to the aroma of many fresh or heated foods (garlic, allium, brassica, onion sprouts, tomatoes, roast meat, potato chips). ${ }^{1-3}$ Alkylthioethanals are generated by oxidative decarboxylation of natural Salkylcysteines (Strecker degradation). ${ }^{1}$ For example, propylthioethanal, possessing a smell of freshly mowed grass, has been prepared as one of the main volatile components, by thermal degradation of equimolar mixtures of S-propyl-L-cysteine and glucose (in $1.34 \%$ yield). ${ }^{3}$

Volatile sulfur compounds are essential for the aroma of many fermented food products like cheese or beverages (wine, beer). ${ }^{4}$ Several unnatural fragrant compositions containing alkylthioethanals have been claimed. ${ }^{5}$ 
The interest in chemistry of 2-alkylthioethanals is sustained by their application in organic synthesis either as carbonyl compounds, ${ }^{6,7}$ or carbanions. ${ }^{8,9,10}$ For instance, alkylthioethanals were previously studied as $\mathrm{CH}$-acids in aldol condensation ${ }^{8}$ (equation 1 , Scheme 1) or in the Mannich reaction performed in water using formaldehyde as methylenating component, ${ }^{9,10}$ (equation 2). When the Mannich reaction proceeds in anhydrous medium in the presence of $\mathrm{CH}_{2} \mathrm{Br}_{2}$ as methylenating reagent, alkylthioethanals 1 show high activity as Michael donors that results in the formation of 2,4-dialkylthiopentanedials as the only products of new domino sequence (equation 3 ) in up to $87 \%$ yield $\left({ }^{1} \mathrm{H}\right.$ NMR). ${ }^{11}$

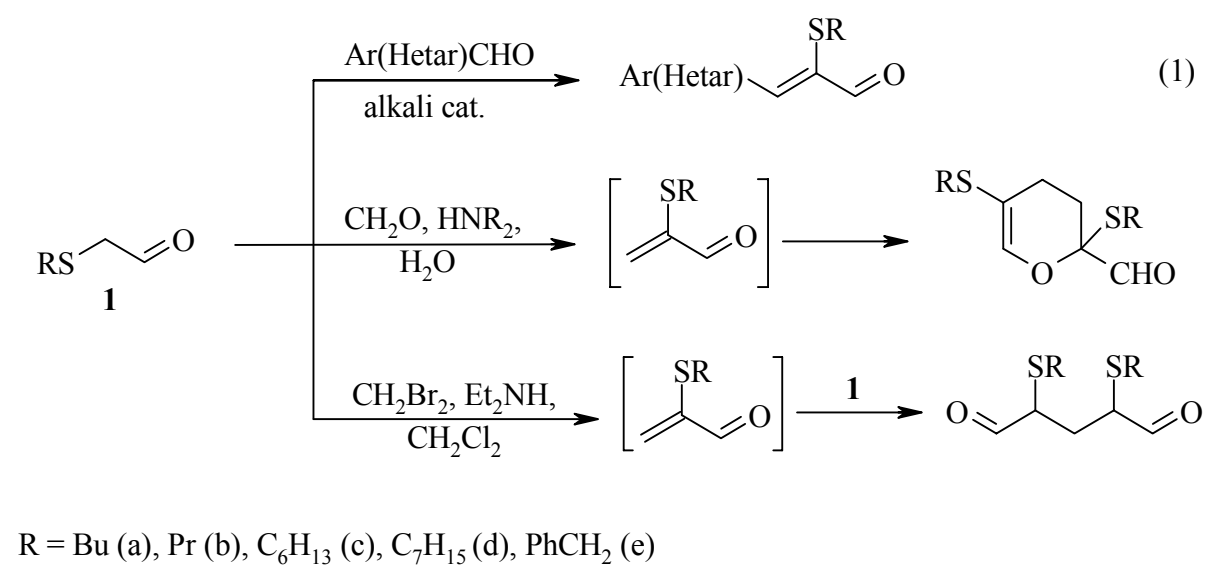

Scheme 1. Synthetic usage of alkylthioethanals.

Alkylthioethanals have found many applications in the synthesis of compounds possessing antihypertensive, ${ }^{12}$ antibacterial, ${ }^{13-15}$ bio-antimutagenic, ${ }^{15}$ fungicide, ${ }^{16}$ and herbicide activities. ${ }^{17}$ They are also employed for the preparation of RS-substituted indoles, ${ }^{18}$ and azapeptides, ${ }^{19}$ to study a proteolytic enzyme associated with Alzheimer's disease.

The yields of alkylthioethanals isolated from natural compounds are negligible. The known protocols for the synthesis of alkylthioethanals concern mainly the aldehydes bearing lower RSradicals (MeS, EtS, BuS). ${ }^{14,20,21,22}$ The target products are often formed in low yields (21$47 \%),{ }^{20,22}$ and synthetic procedures require a strictly limited set of catalysts and solvents, the absence of which leads to the drop of aldehydes yield up to zero. ${ }^{23}$ Recently, a novel method for the synthesis of hexylthioethanal via the reaction of hexyl allyl thioether with $\mathrm{OsO}_{4}$ and $\mathrm{NaIO}_{4}{ }^{15}$ has been reported. However, the former reagent is quite expensive and starting thioether needs to be specially pre-synthesized.

Previously, we obtained alkylthioethanals by the hydrolysis of the corresponding alkylthioacetals, the yields of the target products being 43-59\%. ${ }^{9}$ The purpose of the study was to raise the yields of the reaction and to widen the application area of the protocol for previously unreported high boiling alkylthioethanals, as well as investigate their most typical reaction (sometimes spontaneous). 


\section{Results and Discussion}

In this work, the alkylthioacetal $\mathbf{2}$ hydrolysis selectivity was increased and the yields of alkylthioethanals 1 reached $80-93 \%$ due to the introduction of certain amount of organic solvent, benzene or toluene, into the reaction mixture. This allowed the reaction to be performed at a higher temperature and not only propylthio- and butylthioethanals but also hitherto unknown highly boiling heptylthio-, octylthio- and benzylthioethanals to be prepared in high yields. Besides, usual side reactions accompanying the hydrolysis of acetals $\mathbf{2}$ such as dealcoholysis to ethenes $\mathbf{3}$, aldol condensation of aldehydes $\mathbf{1}$ to enals $\mathbf{4}$ and polymerization were not observed (Scheme 2).

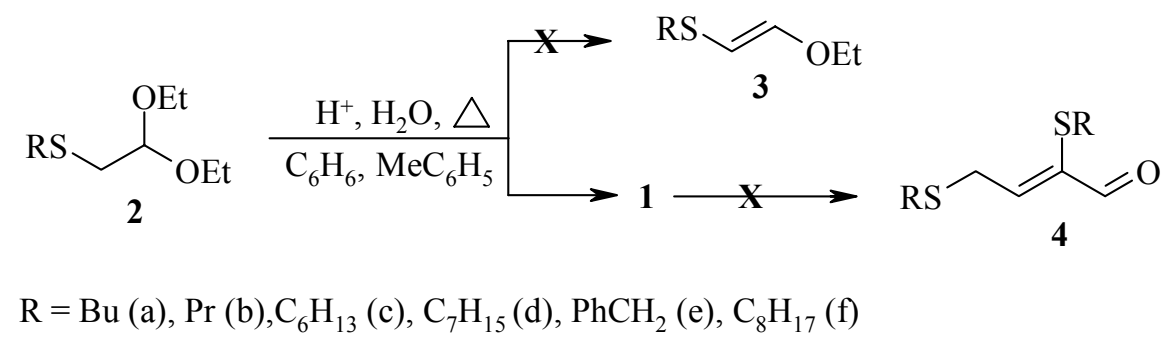

Scheme 2. Synthesis of alkylthioethanals 1 by hydrolysis of their acetals 2.

When butylthioethanal 1a was synthesized via the hydrolysis of its acetal (non-optimized conditions), the aldol self-condensation of aldehyde 1a could reach $28 \%$. The latter process represents a side reaction in cross-aldol condensation, ${ }^{8}$ (equation 1) and in the Mannich reaction, ${ }^{11}$ (equation 3). It was reported that similar self-condensation of ethylthioethanal occurred completely even in the absence of a catalyst on storage of aldehyde during one year without air oxygen and water. ${ }^{20}$ The higher proneness of alkylthioethanals to aldol selfcondensation in acidic or alkaline media prompted us to search for the optimized conditions of direct synthesis of these poorly studied RS-functionalized enals on the example of aldehyde 4a. It turned out that the application of alcohol solutions of MeONa, EtONa or two-phase system $\mathrm{NaOH}-\mathrm{DMF},{ }^{8}$ led to deep resinification of the reaction mixture. When a solution of $1 \mathrm{~N} \mathrm{NaOH}$ was used as a catalyst and the reaction temperature increased up to $70{ }^{\circ} \mathrm{C}$, the yield of aldehyde 4a attained $44 \%\left({ }^{1} \mathrm{H}\right.$ NMR). The employment of $\mathrm{KF}$ in the presence of $\mathrm{Si}(\mathrm{OEt})_{4}$ afforded the target aldehyde $4 \mathrm{a}$ in $60 \%$ yield $\left({ }^{1} \mathrm{H}\right.$ NMR).

A capability of the neat alkylthioethanals $\mathbf{1}$ for spontaneous cyclotrimerization appeared to be a surprising property of them. The works related to cyclotrimerization of aldehydes to the corresponding 2,4,6-trialkyl-1,3,5-trioxanes are abundant in the literature. ${ }^{24}$ The latter compounds find numerous applications in, for example, color photography, ${ }^{25}$ as fumigants, ${ }^{26}$ repellents, deodorants, ${ }^{27}$ and insecticides ${ }^{28}$ Commonly, the synthesis of trioxanes requires usage of the acidic catalysts such as Broensted, ${ }^{29}$ and Lewis, ${ }^{24,30}$ acids, clay, ${ }^{31}$ and ionic liquids,${ }^{24 c, d}$ though spontaneous cyclotrimerization of propanals in water was recently documented. ${ }^{32}$ But 
neither spontaneous nor catalytic cyclotrimerization of alkylthioethanals was previously observed.

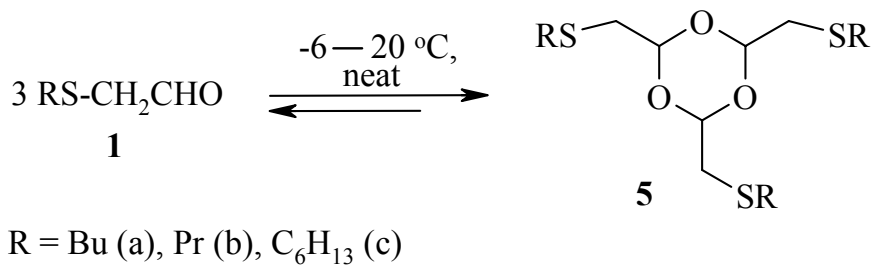

Scheme 3. Trimerization of alkylthioethanals.

We have found for the first time that propylthio-, butylthio- and hexylthioethanals undergo spontaneous cyclotrimerization on storage in refrigerator $\left(-6^{\circ} \mathrm{C}\right)$ without a solvent during from 7 days to several months (Scheme 3). In one of the experiments, propylthioethanal $\mathbf{1 b}$ was almost completely transformed into a trimer $\mathbf{5 b}$ (3 months), which could be easily characterized by ${ }^{1} \mathrm{H}$ NMR technique. In this sample, the signal of carbonyl proton of the initial aldehyde $9.43 \mathrm{ppm}$ has minimal value, while the signal of trimer acetal proton appears as triplet at $5.01 \mathrm{ppm}$ (the signals ratio is $1: 4.4)$. Similar triplets of acetal protons in trimers of ethanal (4.92 ppm), chloroethanal (5.05 ppm), 3-chloropropanal (5.08 ppm), 3-cyanopropanal (5.15 ppm) were observed. ${ }^{24 \mathrm{f}}$ The same picture was observed by us in the ${ }^{1} \mathrm{H}$ NMR spectrum of butylthioethanal 1a (6 months), though the monomer : trimer ratio was $1: 2.3$. The trimerization of hexylthioethanal $1 \mathrm{c}$ made up $21 \%$ on its storage at $-6{ }^{\circ} \mathrm{C}$ for three years in a sealed ampoule. But the ratio trioxane $\mathbf{5 c}$ : aldehyde $\mathbf{1 c}$ in the equilibrium system raised up to $47: 53$ in one month upon keeping hexylthioethanal in a flask at room temperature. Apparently, spontaneous trimerization at lower temperatures can be rationalized by slight oxidation of aldehydes with air oxygen (dissolved in them) to give the corresponding acid, ${ }^{33}$ which catalyzes the trimerization.

Unlike monomers, trimers of aldehydes did not participate in the reactions (1) and (3), performed in alkaline medium. Therefore, the conditions of the starting alkylthioacetaldehydes should be strictly controlled before the application. The trimer of propylthioethanal was detrimerized by distillation in the presence of water and trace amounts of an acid, the yield of the aldehyde reaching $92 \%$.

Previously, on the example of ketones, it was shown that the introduction of sulfur substituent into the $\alpha$-position of the carbonyl moiety increased thermodynamic acidity of a neighbor proton by $\sim 10^{3}$ as compared to simple ketone and stabilized carbon anion adjacent to sulfur atom. ${ }^{34}$ Since the comparative activity of alkylthioacetic aldehydes as $\mathrm{CH}$-acids was not earlier studied, we accomplished the cross-aldol condensation reactions, where furan-2carbaldehyde 6a and thiophene-2-carbaldehyde $\mathbf{6 b}$ acted as carbonyl components and competitive ethoxy- and butylthioethanals played a role of the methylene counterparts (Scheme 4). Two-phase system $\mathrm{NaOH}-\mathrm{DMF}$ was employed as a catalyst. The ratio of the starting reagents $\mathbf{6}: \mathbf{1 a}: \mathbf{7}$ was $1: 1.2: 1.2$. 


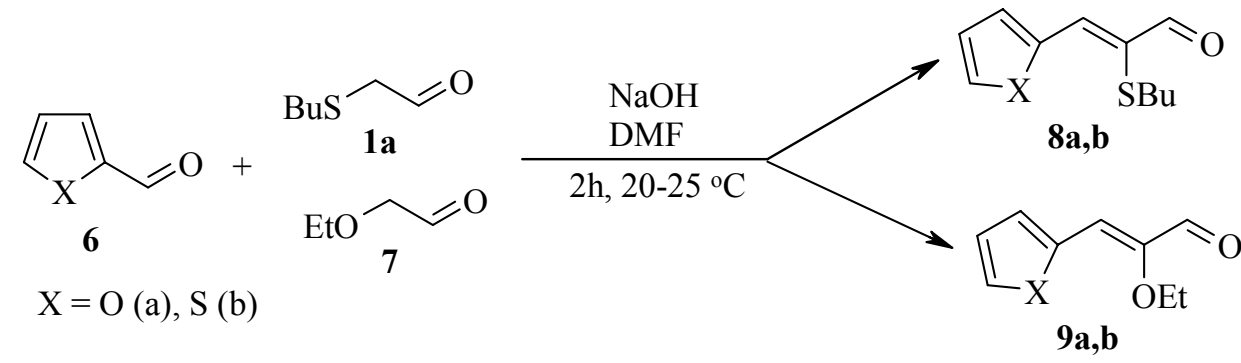

Scheme 4. Synthesis of 2-ethoxy- and 2-alkylthio-3-hetarylpropenals by competitive aldol condensation.

The reaction was controlled using ${ }^{1} \mathrm{H}$ NMR and GC-MS techniques. In the case of aldehyde 6a, the reaction mixture in $2 \mathrm{~h}$ contained products 8a and $9 \mathbf{a}$ in a ratio of $6: 1$ ( ${ }^{1} \mathrm{H} N M R$ ), aldehyde $\mathbf{6 a}$ being completely converted. In the reaction with aldehyde $\mathbf{6} \mathbf{b}$, the conversion of the starting aliphatic aldehydes $(2 \mathrm{~h}$ ) reached 100\%. 2-Butylthio-3-(2-thienyl)propenal $\mathbf{8 b}$ ( $\sim 50 \%)$ was identified as a major reaction product. The expected 2-ethoxy-3-(2-thienyl)propenal $\mathbf{9 b}$ was absent in the reaction mixture that was supported by the comparison of ${ }^{1} \mathrm{H}$ NMR spectral data of mixture with those of authentic sample. To compare spectral data $\left({ }^{1} \mathrm{H}\right.$ NMR and GC-MS), this aldehyde was synthesized by two-component cross-aldol condensation according to the protocol. $^{8}$ Among the reaction products, two compounds with molecular weight 202 ( 13\% of each) were detected (GC-MS). These were likely the products of cross-aldol condensation of the starting aliphatic aldehydes.

\section{Conclusions}

A convenient and effective method is developed to synthesize alkylthioethanals in the yield 80$93 \%$, using commercially available initial reagents. The conditions for their aldol selfcondensation in the presence of basic catalyst have been demonstrated. The spontaneous cyclotrimerization of the neat alkylthioethanals during storing them at the temperature from -6 ${ }^{\circ} \mathrm{C}$ to $20{ }^{\circ} \mathrm{C}$ has been discovered for the first time. Regeneration of the alkylthioethanals out of the corresponding 2,4,6-alkylthiomethyl-1,3,5-trioxanes (in the yield 92\%) is possible by their distillation in the presence of an acid in trace amounts. By the example of cross-aldol condensation, the introduction of a sulfur-containing substituents into $\alpha$-position of the aldehyde group is shown to raise the alkylthioethanals reaction rates as compared with alkoxyethanals by factor of 6 and more. 


\section{Experimental Section}

General. ${ }^{1} \mathrm{H}$ and ${ }^{13} \mathrm{C}$ NMR spectra were recorded on a Bruker DPX 400 spectrometer $(400.13$ and $100.61 \mathrm{MHz}$ accordingly) using $\mathrm{CDCl}_{3}$ as a solvent, and $\mathrm{HMDS}$ as an internal standard. GC - MS analysis was performed using Hewlett-Packard 5971A mass-selective detector (electron impact, $70 \mathrm{eV}$ ) coupled with an HP 5890 gas chromatograph (Ultra-2 column, 5\% of phenylmethylsilicone; injector temperature $250{ }^{\circ} \mathrm{C}$; oven temperature 70 to $280{ }^{\circ} \mathrm{C}$; at rate of 20 ${ }^{\circ} \mathrm{C} \min ^{-1}$ ). IR spectra were recorded in a film on a Bruker Vertex 70 spectrophotometer. Elemental analysis were carried out in a Thermo Finnigan automatic analyzer 1112 ser. Column chromatographic separations were done on silica gel 60 (70-200 mesh).

\section{General procedure for the synthesis of alkylthioethanals (1a-f)}

A mixture of diethyl acetal of alkylthioethanal $2(8.1 \mathrm{mmol})$, water $(144.6 \mathrm{~mL}, 8 \mathrm{~mol})$, conc. $\mathrm{HCl}$ or $p$-toluenesulfonic acid $(70 \pm 4 \mathrm{~mol} \%)$ and $29 \mathrm{~mL}(1 / 5$ of water volume) benzene or toluene was heated at $70{ }^{\circ} \mathrm{C}$ and an intensive stirring during $6-13 \mathrm{~h}$. Then the organic layer was isolated and the aqueous one subjected to a diethyl ether extraction. Organic layers were combined and dried over $\mathrm{MgSO}_{4}$. After removal of ether, the product was isolated by distillation at reduced pressure in nitrogen atmosphere in the presence of hydroquinone. As a result the alkylthioethanals 1 were obtained with the yield $80-93 \%$. Quantities of co-reagents and solvents were proportionally increased to match the amount of initial acetals 2 ranging from 90 to 420 mmol.

Butylthioethanal (1a). Clear liquid with a smell of freshly mowed grass, yield $86 \%, 45.8 \mathrm{~g}$, bp $36-40{ }^{\circ} \mathrm{C} / 1 \mathrm{~mm} \mathrm{Hg}$. IR $\left(v_{\max }, \mathrm{cm}^{-1}\right): 1720$ vs $(\mathrm{C}=\mathrm{O}) .{ }^{1} \mathrm{H}$ NMR $\left(\mathrm{CDCl}_{3}\right): \delta_{\mathrm{H}} 0.91(3 \mathrm{H}, \mathrm{t}, J=7.0$ $\left.\mathrm{Hz}, \mathrm{CH}_{3}\right), 1.39\left(2 \mathrm{H}, \mathrm{m}, \mathrm{CH}_{2}\right), 1.52\left(2 \mathrm{H}, \mathrm{m}, \mathrm{CH}_{2}\right), 2.45\left(2 \mathrm{H}, \mathrm{t}, J=7.0 \mathrm{~Hz}, \mathrm{CH}_{2} \mathrm{~S}\right), 3.15(2 \mathrm{H}, \mathrm{d}, J=$ $\left.3.6 \mathrm{~Hz}, \mathrm{CH}_{2} \mathrm{CHO}\right), 9.43(1 \mathrm{H}, \mathrm{t}, J=3.6 \mathrm{~Hz}, \mathrm{CHO}) .{ }^{13} \mathrm{C} \mathrm{NMR}\left(\mathrm{CDCl}_{3}\right): \delta_{\mathrm{C}} 13.6,21.8,31.2,31.3$, 41.4, 193.6. MS, $m / z(\%)=132\left(\mathrm{M}^{+}, 28\right), 114(6), 103\left(\mathrm{M}^{+}-\mathrm{CHO}, 14\right), 88(15), 61$ (100), 55 (19), 47 (14), 41 (28), 29 (CHO, 19). Anal. Calcd for $\mathrm{C}_{6} \mathrm{H}_{12} \mathrm{OS}$ (132.22): C, 54.50; H, 9.15; S 24.25\%. Found: C, 54.40; H, 9.00; S, 24.00\%.

Propylthioethanal (1b). Clear liquid, yield $81 \%, 40 \mathrm{~g}$, bp 55-60 ${ }^{\circ} \mathrm{C} / 10 \mathrm{~mm} \mathrm{Hg}$. IR $\left(v_{\max }, \mathrm{cm}^{-1}\right)$ : 1720 vs $(\mathrm{C}=\mathrm{O}) .{ }^{1} \mathrm{H}$ NMR $\left(\mathrm{CDCl}_{3}\right): \delta_{\mathrm{H}} 0.98\left(3 \mathrm{H}, \mathrm{t}, J=7.0 \mathrm{~Hz}, \mathrm{CH}_{3}\right), 1.58\left(2 \mathrm{H}, \mathrm{m}, \mathrm{CH}_{2}\right), 2.39$ $\left(2 \mathrm{H}, \mathrm{t}, J=7.0 \mathrm{~Hz}, \mathrm{CH}_{2} \mathrm{~S}\right), 3.13\left(2 \mathrm{H}, \mathrm{d}, J=3.6 \mathrm{~Hz}, \mathrm{CH}_{2} \mathrm{CHO}\right), 9.42(1 \mathrm{H}, \mathrm{t}, J=3.6 \mathrm{~Hz}, \mathrm{CHO}) .{ }^{13} \mathrm{C}$ NMR $\left(\mathrm{CDCl}_{3}\right): \delta_{\mathrm{C}} 13.1,22.3,33.4,41.2,193.3 . \mathrm{MS}, m / z(\%)=118\left(\mathrm{M}^{+}, 47\right), 89\left(\mathrm{M}^{+}-\mathrm{CHO}, 50\right)$, 74 (24), 61 (62), 55 (9), 47 (65), $43\left(\mathrm{CH}_{3}\left(\mathrm{CH}_{2}\right)_{2}, 100\right), 41$ (82). Anal. Calcd for $\mathrm{C}_{5} \mathrm{H}_{10} \mathrm{OS}$ (118.19): C, 50.81; H, 8.53; S, 27.13\%. Found: C, 50.82; H, 8.51; S, 27.00\%.

Hexylthioethanal (1c). ${ }^{9 \mathrm{~b}}$ Yield $77 \%, 10.5 \mathrm{~g}$, bp $84-85{ }^{\circ} \mathrm{C} / 4 \mathrm{~mm} \mathrm{Hg} .{ }^{1} \mathrm{H}$ NMR $\left(\mathrm{CDCl}_{3}\right): \delta_{\mathrm{H}} 0.87$ $\left(3 \mathrm{H}, \mathrm{t}, J=7.0 \mathrm{~Hz}, \mathrm{CH}_{3}\right), 1.30\left(6 \mathrm{H}, \mathrm{m}, \mathrm{CH}_{2}\right), 1.45\left(2 \mathrm{H}, \mathrm{m}, \mathrm{CH}_{2}\right), 2.42\left(2 \mathrm{H}, \mathrm{t}, J=7.3 \mathrm{~Hz}, \mathrm{CH}_{2} \mathrm{~S}\right)$, $3.15\left(2 \mathrm{H}, \mathrm{d}, J=3.6 \mathrm{~Hz}, \underline{\mathrm{H}}_{2} \mathrm{CHO}\right), 9.45(1 \mathrm{H}, \mathrm{t}, J=3.6 \mathrm{~Hz}, \mathrm{CHO}) .{ }^{13} \mathrm{C} \mathrm{NMR}\left(\mathrm{CDCl}_{3}\right): \delta_{\mathrm{C}} 13.9$, 22.5, 28.3, 29.1, 31.2, 31.4, 41.4, 192.9. MS, $m / z(\%)=160\left(\mathrm{M}^{+}, 43\right), 142\left(\mathrm{M}^{+}-\mathrm{H}_{2} \mathrm{O}, 7\right), 131$ ( $\left.\mathrm{M}^{+}-\mathrm{CHO}, 47\right), 117\left(\mathrm{M}^{+}-\mathrm{CH}_{2} \mathrm{CHO}, 22\right), 101$ (7), 87 (31), 83 (67), 75 ( $\left.\mathrm{SCH}_{2} \mathrm{CHO}, 8\right), 69$ (9), 61 
$\left(\mathrm{M}^{+}-\mathrm{CH}_{2} \mathrm{CHO}\right.$ and $\left.\left(\mathrm{CH}_{2}\right)_{4}, 100\right), 55$ (75), 47 (14), 43 (41), 41 (46), 29 (CHO, 21). Anal. Calcd for $\mathrm{C}_{8} \mathrm{H}_{16} \mathrm{OS}$ (160.27): C, 59.95; H, 10.06; S. 20.00\%. Found: C, 59.9; H, 10.13; S. 19.71\%.

Heptylthioethanal (1d). Clear liquid, yield 93\%, $13 \mathrm{~g}$, bp $110{ }^{\circ} \mathrm{C} / 1 \mathrm{~mm} \mathrm{Hg}$. IR $\left(v_{\max }, \mathrm{cm}^{-1}\right)$ : 1722 vs $(\mathrm{C}=\mathrm{O}) .{ }^{1} \mathrm{H}$ NMR $\left(\mathrm{CDCl}_{3}\right): \delta_{\mathrm{H}} 0.88\left(3 \mathrm{H}, \mathrm{t}, J=7.0 \mathrm{~Hz}, \mathrm{CH}_{3}\right), 1.32(6 \mathrm{H}, \mathrm{m}), 1.53(4 \mathrm{H}, \mathrm{m}$, $\left.\mathrm{CH}_{2}\right), 2.40\left(2 \mathrm{H}, \mathrm{t}, J=7.0 \mathrm{~Hz}, \underline{\mathrm{C}}_{2} \mathrm{~S}\right), 3.13\left(2 \mathrm{H}, \mathrm{d}, J=3.5 \mathrm{~Hz}, \underline{\mathrm{C}}_{2} \mathrm{CHO}\right), 9.42(1 \mathrm{H}, \mathrm{t}, J=3.5 \mathrm{~Hz}$, $\mathrm{CHO}) .{ }^{13} \mathrm{C} \mathrm{NMR}\left(\mathrm{CDCl}_{3}\right): \delta_{\mathrm{C}} 14.1,22.6,28.6,28.8,29.1,31.6,31.7,41.3,193.4$.

MS, $m / z(\%)=174\left(\mathrm{M}^{+}, 12\right), 145\left(\mathrm{M}^{+}-\mathrm{CHO}, 15\right), 131\left(\mathrm{M}^{+}-\mathrm{CH}_{2} \mathrm{CHO}, 7\right), 101$ (6), 97 (18), 70 (9), 61 (71), 55 (100), 41 (71). Anal. Calcd for $\mathrm{C}_{9} \mathrm{H}_{18} \mathrm{OS}$ (174.29): C, 62.02; H, 10.41; S, 18.39\%. Found: C, 62.13; H, 10.68; S, 18.41\%.

Benzylthioethanal (1e). Clear liquid, yield 85\%, $24 \mathrm{~g}$, bp 110-120 ${ }^{\circ} \mathrm{C} / 1 \mathrm{~mm} \mathrm{Hg}$. IR $\left(v_{\max }, \mathrm{cm}^{-1}\right)$ : 1717 vs $(\mathrm{C}=\mathrm{O}) .{ }^{1} \mathrm{H}$ NMR $\left(\mathrm{CDCl}_{3}\right): \delta_{\mathrm{H}} 2.98\left(2 \mathrm{H}, \mathrm{d}, J=3.4 \mathrm{~Hz}, \underline{\mathrm{CH}}_{2} \mathrm{CHO}\right), 3.53\left(2 \mathrm{H}, \mathrm{s}, \mathrm{CH}_{2} \mathrm{~S}\right)$, $7.22(5 \mathrm{H}, \mathrm{m}, \mathrm{Ph}), 9.35(1 \mathrm{H}, \mathrm{t}, J=3.4 \mathrm{~Hz}, \mathrm{CHO}) .{ }^{13} \mathrm{C} \mathrm{NMR}\left(\mathrm{CDCl}_{3}\right): \delta_{\mathrm{C}} 35.4,40.1,127.4,128.4$, 128.6, 128.9, 129.1, 136.8, 193.5. MS, $m / z(\%)=166\left(\mathrm{M}^{+}, 18\right), 137\left(\mathrm{M}^{+}-\mathrm{CHO}, 2\right), 122(48), 91$ $\left(\mathrm{C}_{6} \mathrm{H}_{5} \mathrm{CH}_{2}, 100\right), 77$ (4), 65 (13), 45 (6). Anal. Calcd for $\mathrm{C}_{9} \mathrm{H}_{10} \mathrm{OS}$ (166.23): C, 65.02; H, 6.06; S, 19.29\%. Found: C, 65.19; H, 5.96; S, 19.20\%.

Octylthioethanal (1f). Clear liquid, yield $85 \%, 12.1 \mathrm{~g}$, bp $115-119{ }^{\circ} \mathrm{C} / 1 \mathrm{~mm} \mathrm{Hg}$. IR $\left(v_{\max }, \mathrm{cm}^{-}\right.$ $\left.{ }^{1}\right): 1721$ vs $(\mathrm{C}=\mathrm{O}) .{ }^{1} \mathrm{H} \mathrm{NMR}\left(\mathrm{CDCl}_{3}\right): \delta_{\mathrm{H}} 0.88\left(3 \mathrm{H}, \mathrm{t}, J=7.0 \mathrm{~Hz}, \mathrm{CH}_{3}\right), 1.23\left(8 \mathrm{H}, \mathrm{m}, \mathrm{CH}_{2}\right), 1,31$ $\left(2 \mathrm{H}, \mathrm{m}, \mathrm{CH}_{2}\right), 1.54\left(2 \mathrm{H}, \mathrm{m}, \mathrm{CH}_{2}\right), 2.40\left(2 \mathrm{H}, \mathrm{t}, J=7.0 \mathrm{~Hz}, \underline{\mathrm{C}}_{2} \mathrm{~S}\right), 3.14(2 \mathrm{H}, \mathrm{d}, J=3.5 \mathrm{~Hz}$, $\left.\mathrm{C}_{2} \mathrm{CHO}\right), 9.43(1 \mathrm{H}, \mathrm{t}, J=3.5 \mathrm{~Hz}, \mathrm{CHO}) .{ }^{13} \mathrm{C} \mathrm{NMR}\left(\mathrm{CDCl}_{3}\right): \delta_{\mathrm{C}} 14.2,22.7,28.7,29.1,29.2$, 29.3, 31.6, 31.8, 41.4, 193.4. MS, $m / z(\%)=188\left(\mathrm{M}^{+}, 9\right), 170(3), 159\left(\mathrm{M}^{+}-\mathrm{CHO}, 17\right), 145\left(\mathrm{M}^{+}-\right.$ $\left.\mathrm{CH}_{2} \mathrm{CHO}, 9\right), 115$ (4), 101 (14), 87 (18), 70 (11), 69 (100), 61 (80), 55 (66), 41 (77). Anal. Calcd for $\mathrm{C}_{10} \mathrm{H}_{20} \mathrm{OS}$ (188.33): C, 63.77; H, 10.70; S, 17.03\%. Found: C, 64.20; H, 10.80; S, 16.65\%.

\section{General procedure for the synthesis of diethyl acetals of alkylthioethanals (2a-e)}

Sodium (11.5 g, $0.5 \mathrm{~mol}$ ) was added in small portions to $100 \mathrm{~mL}$ of absolute alcohol at stirring. After the complete dissolution of sodium, alkanethiol $(0.5 \mathrm{~mol})$ was added. Diethyl acetal of bromacetaldehyde $(0.5 \mathrm{~mol})$ was added drops wise to the thiolate at heating $\left(70{ }^{\circ} \mathrm{C}\right)$ and an intensive stirring. The heating lasted for $3 \mathrm{~h}$ after that the mixture was kept overnight at room temperature. On the next day, $20 \mathrm{~mL}$ of water was added to the reaction mixture to dissolve $\mathrm{NaBr}$. The organic layer was separated from the aqueous one, which was extracted with diethyl ether. The organic layers were combined and dried over $\mathrm{MgSO}_{4}$. After solvent removal, the product was isolated by vacuum distillation.

Diethyl acetal of butylthioethanal (2a). Clear liquid, yield $81 \%, 83 \mathrm{~g}$, bp $65-80{ }^{\circ} \mathrm{C} / 1 \mathrm{~mm} \mathrm{Hg}$. ${ }^{1} \mathrm{H}$ NMR $\left(\mathrm{CDCl}_{3}\right): \delta_{\mathrm{H}} 0.90\left(3 \mathrm{H}, \mathrm{t}, J=7.3 \mathrm{~Hz}, \mathrm{CH}_{3}(\mathrm{Bu})\right), 1.22\left(6 \mathrm{H}, \mathrm{t}, J=7.0 \mathrm{~Hz}, \mathrm{CH}_{3}(\mathrm{Et})\right), 1.42$ $\left(2 \mathrm{H}, \mathrm{m}, \mathrm{CH}_{2}(\mathrm{Bu})\right), 1.58\left(2 \mathrm{H}, \mathrm{m}, \mathrm{CH}_{2}(\mathrm{Bu})\right), 2.58\left(2 \mathrm{H}, \mathrm{t}, J=7.3 \mathrm{~Hz}, \mathrm{CH}_{2} \mathrm{~S}\right), 2.67(2 \mathrm{H}, \mathrm{d}, J=5.5$ $\left.\mathrm{Hz}, \mathrm{CH}_{2} \mathrm{CH}\right), 3.52\left(2 \mathrm{H}, \mathrm{m}, \mathrm{CH}_{2}(\mathrm{Et})\right), 3.67\left(2 \mathrm{H}, \mathrm{m}, \mathrm{CH}_{2}(\mathrm{Et})\right), 4.56(1 \mathrm{H}, \mathrm{t}, J=5.5 \mathrm{~Hz}, \mathrm{CH}) .{ }^{13} \mathrm{C}$ NMR $\left(\mathrm{CDCl}_{3}\right): \delta_{\mathrm{C}} 13.9,15.5(2 \mathrm{C}), 22.1,31.9,32.7,35.4,62.0(2 \mathrm{C}), 103.5 . \mathrm{MS}, \mathrm{m} / z(\%)=206$ $\left(\mathrm{M}^{+}, 2\right), 161\left(\mathrm{M}^{+}\right.$- OEt, 23), $133\left(\mathrm{M}^{+}-\mathrm{OEt}\right.$ and $\left.\mathrm{CH}_{2} \mathrm{CH}_{2}, 3\right), 103\left(\mathrm{~S}_{-} \mathrm{CH}_{2}-\mathrm{C}-\mathrm{OEt}\right.$ or $\mathrm{CH}(\mathrm{OEt})_{2}$, 82), 75 (82), 61 (21), 47 (100), 41 (20). Anal. Calcd for $\mathrm{C}_{10} \mathrm{H}_{22} \mathrm{O}_{2} \mathrm{~S}$ (206.34): C, 58.21; H, 10.75; S, 15.54\%. Found: C, 57.87; H, 11.12; S, 15.62\%. 
Diethyl acetal of propylthioethanal (2b). Clear liquid, yield $84.4 \%, 81 \mathrm{~g}$, bp $86{ }^{\circ} \mathrm{C} / 10 \mathrm{~mm} \mathrm{Hg}$. ${ }^{1} \mathrm{H}$ NMR $\left(\mathrm{CDCl}_{3}\right): \delta_{\mathrm{H}} 0.98\left(3 \mathrm{H}, \mathrm{t}, J=7.3 \mathrm{~Hz}, \mathrm{CH}_{3}(\mathrm{Pr})\right), 1.20\left(6 \mathrm{H}, \mathrm{t}, J=7.0 \mathrm{~Hz}, \mathrm{CH}_{3}(\mathrm{Et})\right), 1.59$ $\left(2 \mathrm{H}, \mathrm{m}, \mathrm{CH}_{2}(\mathrm{Pr})\right), 2.54\left(2 \mathrm{H}, \mathrm{t}, J=7.3 \mathrm{~Hz}, \mathrm{CH}_{2} \mathrm{~S}\right), 2.63\left(2 \mathrm{H}, \mathrm{d}, J=5.6 \mathrm{~Hz}, \mathrm{CH}_{2} \mathrm{CH}\right), 3.52(2 \mathrm{H}, \mathrm{m}$, $\left.\mathrm{CH}_{2}(\mathrm{Et})\right), 3.65\left(2 \mathrm{H}, \mathrm{m}, \mathrm{CH}_{2}(\mathrm{Et})\right), 4.57(1 \mathrm{H}, \mathrm{t}, J=5.6 \mathrm{~Hz}, \mathrm{CH}) .{ }^{13} \mathrm{C} \mathrm{NMR}\left(\mathrm{CDCl}_{3}\right): \delta_{\mathrm{C}} 13.7,15.6$ (2C), 23.2, 35.1, 35.4, 62.1 (2C), 103.5.MS, $m / z(\%)=192\left(\mathrm{M}^{+}, 1\right), 147\left(\mathrm{M}^{+}-\mathrm{OEt}, 41\right), 119\left(\mathrm{M}^{+}-\right.$ OEt and $\left.\mathrm{CH}_{2} \mathrm{CH}_{2}, 11\right), 103$ (S-CH$-\mathrm{C}-\mathrm{OEt}$ or $\left.\mathrm{CH}(\mathrm{OEt})_{2}, 100\right), 89$ (7), 75 (64), 61 (7), 47 (58), 41 (9), 29 (11). Anal. Calcd for $\mathrm{C}_{9} \mathrm{H}_{20} \mathrm{O}_{2} \mathrm{~S}$ (192.31): C, 56.21; H, 10.48; S, 16.67\%. Found: C, 56.09; H, 10.56; S, 16.74\%.

Diethyl acetal of hexylthioethanal (2c). Clear liquid, yield $88 \%, 93 \mathrm{~g}$, bp $122{ }^{\circ} \mathrm{C} / 1 \mathrm{~mm} \mathrm{Hg} .{ }^{1} \mathrm{H}$ $\operatorname{NMR}\left(\mathrm{CDCl}_{3}\right): \delta_{\mathrm{H}} 0.88\left(3 \mathrm{H}, \mathrm{t}, J=7.0 \mathrm{~Hz}, \mathrm{CH}_{3}\right), 1.22\left(6 \mathrm{H}, \mathrm{t}, J=7.0 \mathrm{~Hz}, \mathrm{CH}_{3}(\mathrm{Et})\right), 1.29(4 \mathrm{H}, \mathrm{m}$, $\left.\mathrm{CH}_{2}\right), 1.37\left(2 \mathrm{H}, \mathrm{m}, \mathrm{CH}_{2}\right), 1.55\left(2 \mathrm{H}, \mathrm{m}, \mathrm{CH}_{2}\right), 2.58\left(2 \mathrm{H}, \mathrm{t}, J=7.0 \mathrm{~Hz}, \mathrm{CH}_{2} \mathrm{CH}_{2} \mathrm{~S}\right), 2.68(2 \mathrm{H}, \mathrm{d}, J=$ $\left.5.5 \mathrm{~Hz}, \mathrm{SCH}_{2} \mathrm{CHO}\right), 3.55\left(2 \mathrm{H}, \mathrm{m}, \mathrm{CH}_{2}(\mathrm{Et})\right), 3.68\left(2 \mathrm{H}, \mathrm{m}, \mathrm{CH}_{2}(\mathrm{Et})\right), 4.60(1 \mathrm{H}, \mathrm{t}, J=5.5 \mathrm{~Hz}, \mathrm{CH})$. ${ }^{13} \mathrm{C} \mathrm{NMR}\left(\mathrm{CDCl}_{3}\right): \delta_{\mathrm{C}} 14.0,15.3(2 \mathrm{C}), 22.5,28.5,29.6,31.4,32.8,35.3,61.89(2 \mathrm{C})$, 103.3. MS, $m / z(\%)=234\left(\mathrm{M}^{+}, 1\right), 189\left(\mathrm{M}^{+}-\mathrm{OEt}, 31\right), 161\left(\mathrm{M}^{+}-\mathrm{OEt}\right.$ and $\left.\left(\mathrm{CH}_{2}\right)_{2}, 2\right), 103\left(\mathrm{~S}_{-} \mathrm{CH}_{2}-\mathrm{C}-\mathrm{OEt}\right.$ or $\left.\mathrm{CH}(\mathrm{OEt})_{2}, 100\right), 85$ (5), 75 (S- $\left.\mathrm{CH}_{2}-\mathrm{C}-\mathrm{OH}, 52\right), 47$ (31), 43 (14), 29 (CHO, 9). Anal. Calcd for $\mathrm{C}_{12} \mathrm{H}_{26} \mathrm{O}_{2} \mathrm{~S}$ (234.39): C, 61.49; H, 11.18; S, 13.68\%. Found: C, 61.60; H, 11.29; S, 13.72\%.

Diethyl acetal of heptylthioethanal (2d). Clear liquid, yield $92 \%, 106.5 \mathrm{~g}, \mathrm{bp} 128{ }^{\circ} \mathrm{C} / 1 \mathrm{~mm} \mathrm{Hg}$. ${ }^{1} \mathrm{H}$ NMR $\left(\mathrm{CDCl}_{3}\right): \delta_{\mathrm{H}} 0.88\left(3 \mathrm{H}, \mathrm{t}, J=7.0 \mathrm{~Hz}, \mathrm{CH}_{3}\right), 1.20\left(6 \mathrm{H}, \mathrm{t}, J=7.0 \mathrm{~Hz}, \mathrm{CH}_{3}(\mathrm{Et})\right), 1.34(8 \mathrm{H}$, m, heptyl), $1.58\left(2 \mathrm{H}, \mathrm{m}, \mathrm{CH}_{2}\right.$, heptyl), $2.55\left(2 \mathrm{H}, \mathrm{t}, J=7.0 \mathrm{~Hz}, \mathrm{CH}_{2} \mathrm{~S}\right), 2.63(2 \mathrm{H}, \mathrm{d}, J=5.5 \mathrm{~Hz}$, $\left.\mathrm{C}_{2} \mathrm{CH}\right), 3.50\left(2 \mathrm{H}, \mathrm{m}, \mathrm{CH}_{2}(\mathrm{Et})\right), 3.64 \mathrm{м}\left(2 \mathrm{H}, \mathrm{m}, \mathrm{CH}_{2}(\mathrm{Et})\right), 4.55(1 \mathrm{H}, \mathrm{t}, J=5.5 \mathrm{~Hz}, \mathrm{CH}) .{ }^{13} \mathrm{C}$ $\operatorname{NMR}\left(\mathrm{CDCl}_{3}\right): \delta_{\mathrm{C}} 14.0,15.3(2 \mathrm{C}), 22.6,28.8,28.9,29.6,31.7,32.8,35.2,61.8(2 \mathrm{C})$, 103.3. MS, $m / z(\%)=203\left(\mathrm{M}^{+}\right.$- OEt, 26), $103\left(\mathrm{~S}-\mathrm{CH}_{2}-\mathrm{C}-\mathrm{OEt}\right.$ or $\left.\mathrm{CH}(\mathrm{OEt})_{2}, 100\right), 75$ (52), 57 (13), 47 (37), 29 (14). Anal. Calcd for $\mathrm{C}_{13} \mathrm{H}_{28} \mathrm{O}_{2} \mathrm{~S}$ (248.41): C, 62.85; H, 11.36; S, 12.91\%. Found: C, 63.23; H, $11.31 ; \mathrm{S}, 12.90 \%$.

Diethyl acetal of benzylthioethanal (2e). Clear liquid, yield $92 \%, 110 \mathrm{~g}$, bp $130{ }^{\circ} \mathrm{C} / 1 \mathrm{~mm} \mathrm{Hg}$. ${ }^{1} \mathrm{H}$ NMR $\left(\mathrm{CDCl}_{3}\right): \delta_{\mathrm{H}} 1.20\left(6 \mathrm{H}, \mathrm{t}, J=7.0 \mathrm{~Hz}, \mathrm{CH}_{3}\right), 2.57\left(2 \mathrm{H}, \mathrm{d}, J=5.5 \mathrm{~Hz}, \mathrm{C}_{2} \mathrm{CH}\right), 3.52(2 \mathrm{H}$, $\left.\mathrm{m}, \mathrm{OCH}_{2}\right), 3.65\left(2 \mathrm{H}, \mathrm{m}, \mathrm{OCH}_{2}\right), 3.78\left(2 \mathrm{H}, \mathrm{s}, \mathrm{CH}_{2} \mathrm{~S}\right), 4.53(1 \mathrm{H}, \mathrm{t}, J=5.5 \mathrm{~Hz}, \mathrm{CH}), 7.20(5 \mathrm{H}, \mathrm{m}$, $\mathrm{Ph}) .{ }^{13} \mathrm{C}$ NMR $\left(\mathrm{CDCl}_{3}\right): \delta_{\mathrm{C}} 15.3(2 \mathrm{C}), 34.0,36.6,61.9(2 \mathrm{C}), 103.3,126.8,128.3(2 \mathrm{C}), 129.0$ (2C), 138.4. MS, $m / z(\%)=194\left(\mathrm{M}^{+}\right.$- OEt and H, 8), 149 (20), 122 (6), $103\left(\mathrm{~S}_{-} \mathrm{CH}_{2}-\mathrm{C}-\mathrm{OEt}\right.$ or $\left.\mathrm{CH}(\mathrm{OEt})_{2}, 100\right), 91$ (87), 75 (54), 65 (15), 47 (51), 29 (13). Anal. Calcd for $\mathrm{C}_{13} \mathrm{H}_{20} \mathrm{OS}$ (224.39): C, 64.96; H, 8.39; S, 13.34\%. Found: C, 65.20; H, 8.29; S, 12.97\%.

Diethyl acetal of octylthioethanal (2f). Clear liquid, yield $92 \%, 214 \mathrm{~g}$, bp $131{ }^{\circ} \mathrm{C} / 1 \mathrm{~mm} \mathrm{Hg} .{ }^{1} \mathrm{H}$ $\operatorname{NMR}\left(\mathrm{CDCl}_{3}\right): \delta_{\mathrm{H}} 0.88\left(3 \mathrm{H}, \mathrm{t}, J=7.0 \mathrm{~Hz}, \mathrm{CH}_{3}\right), 1.21\left(6 \mathrm{H}, \mathrm{t}, J=7.0 \mathrm{~Hz}, \mathrm{CH}_{3}(\mathrm{Et})\right), 1.23(10 \mathrm{H}, \mathrm{m}$, octyl), $1.57\left(2 \mathrm{H}, \mathrm{m}\right.$, octyl), $2.56\left(2 \mathrm{H}, \mathrm{t}, J=7.3 \mathrm{~Hz}, \mathrm{CH}_{2} \mathrm{~S}\right), 2.65\left(2 \mathrm{H}, \mathrm{d}, J=5.5 \mathrm{~Hz}, \mathrm{CH}_{2} \mathrm{CH}\right), 3.53$ $\left(2 \mathrm{H}, \mathrm{m}, \mathrm{CH}_{2}(\mathrm{Et})\right), 3.65 \mathrm{~m}\left(2 \mathrm{H}, \mathrm{m}, \mathrm{CH}_{2}(\mathrm{Et})\right), 4.56(1 \mathrm{H}, \mathrm{t}, J=5.5 \mathrm{~Hz}, \mathrm{CH}) .{ }^{13} \mathrm{C} \mathrm{NMR}\left(\mathrm{CDCl}_{3}\right): \delta_{\mathrm{C}}$ $14.1,15.4$ (2C), 22.7, 28.9, 29.2, 29.3, 29.7, 31.9, 32.9, 35.3, 61.9 (2C), 103.4. MS, $m / z(\%)=$ $217\left(\mathrm{M}^{+}\right.$- OEt, 17), 103 (S-CH $2-\mathrm{C}-\mathrm{OEt}$ or $\left.\mathrm{CH}(\mathrm{OEt})_{2}, 100\right), 75$ (43), 55 (7), 47 (26), 29 (8). Anal. Calcd for $\mathrm{C}_{14} \mathrm{H}_{30} \mathrm{O}_{2} \mathrm{~S}$ (262.44): C, 64.07; H, 11.52; S, 12.22\%. Found: C, 63.91; H, 11.43; S, $12.33 \%$. 
Hydrolysis of dimethyl acetal of butylthioethanal (non-optimized conditions). A mixture of diethyl acetal of butylthioethanal $(129.8 \mathrm{~g}, 0.73 \mathrm{~mol}), \mathrm{H}_{2} \mathrm{O}(300 \mathrm{~mL}, 16.7 \mathrm{~mol}), 2 \mathrm{~mL}$ concentrated hydrochloric acid and toluene $(400 \mathrm{~mL})$ was refluxed and stirred for $4 \mathrm{~h}$. Than the organic layer was decanted. The water layer was extracted with ether $(5 \times 20 \mathrm{~mL})$. The organic layers were combined and dried over anhydrous $\mathrm{MgSO}_{4}$. After removal of ether, the residue was distillated in high vacuum. There was obtaned $30 \mathrm{~g}(32 \%)$ butylthioethanal 1a bp $50{ }^{\circ} \mathrm{C} / 2 \mathrm{~mm}$ $\mathrm{Hg}$ and $25.4 \mathrm{~g} \mathrm{(28 \% )}$ of 2,4-dibutylthio-2-butenal 4, bp $162{ }^{\circ} \mathrm{C} / 2 \mathrm{~mm} \mathrm{Hg}$. Its ${ }^{1} \mathrm{H}$ and ${ }^{13} \mathrm{C}$ spectra are identical to the ones of authentic sample described below.

2,4-Dibutylthio-2-butenal (4). The butylthioethanal (13.2 g, $100 \mathrm{mmol})$ was added slowly dropwise to a stirred mixture of $\mathrm{KF}(2.9 \mathrm{~g}, 0.05 \mathrm{mmol})$ and $\mathrm{Si}(\mathrm{OEt})_{4}(5.6 \mathrm{~mL})$ in EtOH $(41 \mathrm{~mL})$ and stirred for $1.5 \mathrm{~h}$ at $0{ }^{\circ} \mathrm{C}$. The reaction mixture was diluted with water and extracted with ether $(5 \times 10 \mathrm{~mL})$. Organic extract was dried over $\mathrm{MgSO}_{4}$. After removal of solvents, the product was isolated by vacuum distillation, bp $162{ }^{\circ} \mathrm{C} / 2 \mathrm{~mm} \mathrm{Hg}$. Yield $28 \%, 2.8 \mathrm{~g}$, clear yellow oil. ${ }^{1} \mathrm{H}$ NMR $\left(\mathrm{CDCl}_{3}\right): \delta_{\mathrm{H}} 0.90$ and $0.92\left(6 \mathrm{H}\right.$, two t, $\left.{ }^{3} J=7.3 \mathrm{~Hz}, \mathrm{CH}_{3}\right), 1.42\left(4 \mathrm{H}, \mathrm{m}, \mathrm{C}_{2} \mathrm{CH}_{3}\right), 1.58(4 \mathrm{H}$, m, $\left.\mathrm{CH}_{2} \mathrm{CH}_{2} \mathrm{CH}_{3}\right), 2.45\left(2 \mathrm{H}, \mathrm{t},{ }^{3} J=7.4 \mathrm{~Hz}, \mathrm{SCH}_{2}\right), 2.83\left(2 \mathrm{H}, \mathrm{t},{ }^{3} J=7.3 \mathrm{~Hz}, \mathrm{SCH}_{2}\right), 3.58(2 \mathrm{H}, \mathrm{d}, J$ $\left.=7.7 \mathrm{~Hz}, \mathrm{CH}_{2}\right), 6.87(1 \mathrm{H}, \mathrm{t}, J=7.7 \mathrm{~Hz},=\mathrm{CH}), 9.46(1 \mathrm{H}, \mathrm{s}, \mathrm{CHO}) .{ }^{13} \mathrm{C} \mathrm{NMR}\left(\mathrm{CDCl}_{3}\right): \delta_{\mathrm{C}} 13.5$, 13.7, 21.3, 22.0, 23.3, 29.1, 31.9, 32.6, 33.9, 138.6, 140.9, 185.4. MS, $m / z(\%)=246\left(\mathrm{M}^{+}, 42\right)$ $217\left(\mathrm{M}^{+}-\mathrm{Et}, 18\right), 189\left(\mathrm{M}^{+}-\mathrm{Bu}, 22\right), 156\left(\mathrm{M}^{+}-\mathrm{BuSH}, 33\right), 133$ (32), 113 (17), 100 (M+BuSH$\left.\left(\mathrm{CH}_{2}\right)_{4}, 86\right), 99$ (100), 87 (14), 73 (20), 71 (58), $57\left(\mathrm{Bu}^{+}, 26\right)$, 41(43). Anal. Calcd for $\mathrm{C}_{12} \mathrm{H}_{22} \mathrm{OS}_{2}$ (246.42): C, 58.49; H, 9.00; S, 26.02\%. Found: C, 58.42; H, 8.87; S, 26.10\%.

Spontaneous trimerization of alkylthioethanals (1a-c). The corresponding neat aldehydes 1a, $\mathbf{b}, \mathbf{c}$ was allowed to stand in a tightly stoppered flask or ampoule at the $-6^{\circ} \mathrm{C}$ or r.t. for several days or months, the reaction progress being monitored by ${ }^{1} \mathrm{H}$ NMR.

2,4,6-Butylthiomethyl-1,3,5-trioxane (5a). This compound was found to form in mixture in $70 \%$ yield when butylthioethanal (1a) was kept at $-6{ }^{\circ} \mathrm{C}$ for 1.5 months. ${ }^{1} \mathrm{H}$ NMR $\left(\mathrm{CDCl}_{3}\right): \delta_{\mathrm{H}}$ $0.91\left(9 \mathrm{H}, \mathrm{t}, J=7.2 \mathrm{~Hz}, \mathrm{CH}_{3}\right), 1.39\left(6 \mathrm{H}, \mathrm{m}, \mathrm{CH}_{3} \mathrm{C}_{2}\right), 1.53\left(6 \mathrm{H}, \mathrm{m}, \mathrm{C}_{2} \mathrm{CH}_{2} \mathrm{~S}\right), 2.60(6 \mathrm{H}, \mathrm{t}, J=$ $\left.7.4 \mathrm{~Hz}, \mathrm{SCH}_{2}\right), 2.72\left(6 \mathrm{H}, \mathrm{d}, J=5.0 \mathrm{~Hz}, \mathrm{C}_{2} \mathrm{CH}\right), 5.04(3 \mathrm{H}, \mathrm{t}, J=5.0 \mathrm{~Hz}, \mathrm{OCHO}) .{ }^{13} \mathrm{C} \mathrm{NMR}$ $\left(\mathrm{CDCl}_{3}\right): \delta_{\mathrm{C}} 13.7,21.9,31.6,32.8,35.1,102.1$.

2,4,6-Propylthiomethyl-1,3,5-trioxane (5b). This compound was observed to form in $81 \%$ yield when propylthioethanal (1b) was kept at $-6{ }^{\circ} \mathrm{C}$ for 3 months. ${ }^{1} \mathrm{H}$ NMR $\left(\mathrm{CDCl}_{3}\right)$ : $\delta_{\mathrm{H}} 0.98$ $\left(9 \mathrm{H}, \mathrm{t}, J=7.2 \mathrm{~Hz}, \mathrm{CH}_{3}\right), 1.59\left(6 \mathrm{H}, \mathrm{m}, \mathrm{CH}_{3} \mathrm{C}_{2}\right), 2.57\left(6 \mathrm{H}, \mathrm{t}, J=7.2 \mathrm{~Hz}, \mathrm{SCH}_{2}\right), 2.69(6 \mathrm{H}, \mathrm{d}, J=$ $\left.5.1 \mathrm{~Hz}, \underline{\mathrm{C}}_{2} \mathrm{CH}\right), 5.02(3 \mathrm{H}, \mathrm{t}, J=5.1 \mathrm{~Hz}, \mathrm{OCHO})$. The compound was resinified in attempting to isolate by vacuum distillation in nitrogen atmosphere.

2,4,6-Hexylthiomethyl-1,3,5-trioxane (5c). This compound was observed in $21 \%$ yield when hexylthioethanal $(\mathbf{1 c})$ was allowed to stand in ampoule for 3 years at $-6{ }^{\circ} \mathrm{C} .{ }^{1} \mathrm{H}$ NMR $\left(\mathrm{CDCl}_{3}\right): \delta_{\mathrm{H}}$ $0.88\left(9 \mathrm{H}, \mathrm{t}, J=7.0 \mathrm{~Hz}, \mathrm{CH}_{3}\right), 1.28\left(6 \mathrm{H}, \mathrm{m}, \mathrm{CH}_{2}\right), 1.36\left(12 \mathrm{H}, \mathrm{m},\left(\mathrm{CH}_{2}\right)_{2}\right), 1.53\left(12 \mathrm{H}, \mathrm{m},\left(\mathrm{CH}_{2}\right)_{2}\right)$, $2.58\left(6 \mathrm{H}, \mathrm{m}, \mathrm{SCH}_{2}\right), 2.72\left(6 \mathrm{H}, \mathrm{d}, J=5.2 \mathrm{~Hz}, \mathrm{C}_{2} \mathrm{CH}\right), 5.03(3 \mathrm{H}, \mathrm{t}, J=5.2 \mathrm{~Hz}, \mathrm{OCHO}) .{ }^{13} \mathrm{C}$ $\operatorname{NMR}\left(\mathrm{CDCl}_{3}\right): \delta_{\mathrm{C}} 14.1,22.5,28.5,28.8,29.4,31.4,35.1,102.1$. 
Detrimerization of 2,4,6-propylthiomethyl-1,3,5-trioxane (5b). A mixture $10 \mathrm{~g}(84.7 \mathrm{mmol})$ propylthioethanal, $5 \mathrm{~mL}(0.28 \mathrm{~mol}) \mathrm{H}_{2} \mathrm{O}$ and $0.1 \mathrm{~g}(0.53 \mathrm{mmol}) p$-TsOH was stirred for $25 \mathrm{~min}$ at $25{ }^{\circ} \mathrm{C}$. Then it was distilled in vacuum, bp $55-60{ }^{\circ} \mathrm{C} / 10 \mathrm{~mm} \mathrm{Hg}$, the yield of the aldehyde $\mathbf{1 b}$ $92 \%, 9.2 \mathrm{~g}$.

\section{Synthesis of 2-ethoxy- and 2-alkylthio-3-hetarylpropenals by competitive aldol condensation}

Interaction of furan-2-carbaldehyde with ethoxy- and butylthioethanals. Furan-2carbaldehyde $(0.3 \mathrm{~g}, 3.1 \mathrm{mmol})$ was added at once to a continuously stirred mixture of $\mathrm{NaOH}$ $(0.03 \mathrm{~g}, 0.75 \mathrm{mmol})$ and DMF $(12 \mathrm{~mL})$ at room temperature. Then a solution of butylthioethanal $(0.49 \mathrm{~g}, 3.72 \mathrm{mmol})$ in $5 \mathrm{~mL}$ DMF and a solution of ethoxyethanal $(0.33 \mathrm{~g}, 3.72 \mathrm{mmol})$ in $5 \mathrm{~mL}$ DMF were added simultaneously drops wise. The mixture stirring was continued for $2 \mathrm{~h}$ at room temperature, after that the mixture was diluted with water and extracted with benzene. Organic layer was washed with water $(3 \times 5 \mathrm{~mL})$ to remove DMF and dried over $\mathrm{MgSO}_{4}$. After removal of benzene in vacuum, the mixture of two products was obtained. It consisted of 2-butylthio-3(2-furyl)propenal 8a and 3-(2-furyl)-2-ethoxypropenal 9a in a ratio 6: 1. Their ${ }^{1} \mathrm{H}$ NMR spectra were identical to those of the authentical samples. ${ }^{8}$

Interaction of thiophen-2-carbaldehyde with ethoxy- and butylthioethanals. The reaction was performed in a similar manner as above starting from thiophen-2-carbaldehyde (1.78 mmol) and of ethoxy- and butylthioethanals (by $2.14 \mathrm{mmol}$ ). After removal of benzene in vacuum, the mixture was shown to contain three new compounds and the initial thiophen-2-carbaldehyde $6 \mathbf{b}$ (by ${ }^{1} \mathrm{H}$ NMR). According to GC-MS, the reaction mixture contained 2-butylthio-3-(2thienyl)propenal $\mathbf{8 b}$, and the products of cross aldol condensation of aliphatic aldehydes (4butylthio-2-ethoxybutenal and 2-butylthio-4-ethoxybutenal) in a ratio $2: 1: 1$ respectively. ${ }^{1} \mathrm{H}$ and ${ }^{13} \mathrm{C}$ spectra of aldehyde $\mathbf{8 b}$ are identical to the published ones of authentic sample. ${ }^{8}$ To prove total absence of potential aldehyde $\mathbf{9 b}$ in the reaction mixture, it was obtained by two-component reaction by protocol. ${ }^{8}$

(Z)-3-(2-Thienyl)-2-ethoxypropenal (9b). Was obtained by the protocol. ${ }^{8}$ Clear dark-orange liquid, yield 42\%, $1.4 \mathrm{~g}$ after column chromatography on silica gel using hexane / ether $3: 1 .{ }^{1} \mathrm{H}$ $\operatorname{NMR}\left(\mathrm{CDCl}_{3}\right): \delta_{\mathrm{H}} 1.42\left(3 \mathrm{H}, \mathrm{t}, J=7.1 \mathrm{~Hz}, \mathrm{CH}_{3}\right), 4.33\left(2 \mathrm{H}, \mathrm{q}, J=7.1 \mathrm{~Hz}, \mathrm{OCH}_{2}\right), 6.91(1 \mathrm{H}, \mathrm{s},=\mathrm{CH})$, $7.09(1 \mathrm{H}, \mathrm{dd}, J=5.1 \mathrm{~Hz}, J=3.7 \mathrm{~Hz}, \mathrm{H}-4), 7.35(1 \mathrm{H}, \mathrm{d}, J=3.7 \mathrm{~Hz}, \mathrm{H}-3), 7.51(1 \mathrm{H}, \mathrm{d}, J=5.1 \mathrm{~Hz}$, $\mathrm{H}-5), 9.30(1 \mathrm{H}, \mathrm{s}, \mathrm{CHO}) .{ }^{13} \mathrm{C} \mathrm{NMR}\left(\mathrm{CDCl}_{3}\right): \delta_{\mathrm{C}} 15.8,67.3,127.2,128.2,131.0,131.5,136.3$, 143.2, 187.8. MS, $m / z(\%)=182\left(\mathrm{M}^{+}, 36\right), 154\left(\mathrm{M}^{+}-\mathrm{CH}_{2}=\mathrm{CH}_{2}, 9\right), 138$ (40), 125 (48), 110 (6), 97 (100), $84\left(\mathrm{C}_{4} \mathrm{H}_{4} \mathrm{~S}, 6\right), 70$ (12), 53 (9), 45 (OEt, 18), 39 (9). Anal. Calcd for $\mathrm{C}_{9} \mathrm{H}_{10} \mathrm{O}_{2} \mathrm{~S}$ (182.23): C, 59.32; H, 5.53; S, 17.59\%. Found: C, 59.80; H, 5.58; S, 17.44\%.

\section{References}

1. Rössner, J.; Kubec, R.; Velišek, J.; Davidek, J. Eur. Food Res. Technol. 2002, $215,124$. 
2. (a) Takahashi, M.; Shibamoto, T. J. Agric. Food. Chem. 2008, 56, 10462. (b) Birtic, S.; Ginies, C.; Cause, M.; Renard, C. M.; Page, D. J. Agric. Food. Chem. 2009, 57, 591. (c) Rochat, S.; de Saint Laumer, J.-Y.; Chaintreau, A. J. Chromatography A. 2007, 1147 (1), 85. (d) Kubec, R.; Drohova, V.; Velišek, J. Agric. Food. Chem. 1999, 47, 1132. (e) Buttery, R. G.; Ling, L. C. J. Agric. Food. Chem. 1972, 20, 698. (f) Buttery, R. G.; Seifert, R. M.; Guadagni, D. G.; Ling, L. C. J. Agric. Food. Chem. 1971, 19, 524.

3. Kimura, K.; Jvata, I.; Nishimura, H.; Kasuya, M.; Yamane, A.; Mizutani, J. Agric. Biol. Chem. 1990, 54, 1893.

4. Landaud, S.; Helinck, S.; Bonnarme, P. Appl. Microbiol. Biotech. 2008, 77(6), 1191.

5. (a) Kawasaki K. Japan Pat. 2006124490, 2006; Chem. Abstr. 2006, 144, 467073. (b) Kawasaki K. Japan Pat. 2005013138, 2005; Chem. Abstr. 2005, 142, 133471. (c) Kawasaki K. Japan Pat. 2005015684, 2005; Chem. Abstr. 2005, 142, 133468.

6. (a) Sorokin, M. S.; Lopyrev, V. A.; Voronkov M. G. J. Gen. Chem. (Russ.). 2005, 75, 945; Chem. Abstr. 2006, 144, 488296. (b) Frost, C. G.; Williams, J. M. J. Synlett 1994, 551. (c) Bałczewski, P.; Mikołajczyk, M. Zeit. für Naturforschung, B 1989, 44. 99.

7. (a) Kudzin, Z. H. Synthesis. 1981, 643. (b) Pittet, A. O.; Courtney, T. F.; Vock, M. H.; Bowen, D. R.; Muralidhara, R. US. Patent 4472 446, 1984; Chem. Abstr. 1985, 102, 23203.

8. Keiko, N. A.; Stepanova, L. G.; Verochkina, E. A.; Larina, L. I. Arkivoc 2010, (ii), 49.

9. (a) Keiko, N. A.; Stepanova, L. G. Izv. Acad. Nauk SSSR, Ser. Khim. (Russ.). 1972, 2516; Chem. Abstr. 1973, 78, 71838. (b) Keiko, N. A.; Stepanova, L. G.; Kalikhman, I. D.; Voronkov, M. G. Izv. Acad. Nauk SSSR, Ser. Khim. (Russ.). 1977, 1652; Chem. Abstr. 1977, 151955.

10. Keiko, N. A.; Chuvashev, Yu. A.; Stepanova, L. G.; Bannikova, O. B.; Voronkov, M. G. Izv. Acad. Nauk SSSR, Ser. Khim. (Russ.). 1996, 188; Chem. Abstr. 1996, 124, 316476.

11. Keiko, N. A.; Stepanova, L. G.; Verochkina, E. A.; Larina, L. I.; Chuvashev, Yu. A. Arkivoc 2008, (xvii), 184.

12. Kujath, E.; Baumgartner, C.; Schönafinger, K.; Beyerle, R.; Just, M.; Bohn, H.; Ostrowski, J. Patent DE. 3921 460, 1991; Chem. Abstr. 1991, 114, 122388.

13. (a) Stepanova, L. G.; Keiko, N.A.; Malkova, T. N.; Leont'eva, A. G.; Pushechkina, T. Ya.; Voronkov, M. G. Khim. Pharm. Zh. (Russ.). 1988, 710; Chem. Abstr. 1989, 110, 38839. (b) Keiko, N. A.; Stepanova, L. G.; Kleptsova, E. A.; Vdovina, G. P.; Odegova, T. F. Khim. Pharm. Zh. (Russ.). 2009, 43(9), 502; Chem. Abstr. 2010, 152, 168552. (c) Tamura, N.; Natsugari, H.; Kawano, Y.; Matsushita, Y.; Yoshioka, K.; Ochiai, M. Chem. Pharm. Bull. 1987, 35, 996. (d) Yoshicka, K.; Tamura, N.; Natsugari, H. Eur.Pat. 137403, 1985; Chem. Abstr. 1996, 125 , 301026.

14. Micołajchyk, M.; Bałczewski, P. Synthesis 1987, 659.

15. Takai, K.; Nakamura, A.; Shibutani, K.; Iriye, R. Biocontrol Science. 2002, 7, 111.

16. Volchatova, I. V.; Medvedeva, C. A.; Keiko, N. A. Izv. Vissh. Ucheb. Zaved. Stroitelstvo (Russ.). 2001, 12, 47.

17. Lee, L. F. Eur.Pat. 135 491, 1985; Chem. Abstr. 1985, 103, 178173.

18. Gassman, P. G.; van Bergen, T. J.; Gilbert, D. P.; Cue, B. W. J. Am. Chem. Soc. 1974, 96, 5495. 
19. Quibell, M.; Turnell, W. G.; Johnson, T. J. Chem. Soc., Perkin Trans. 1 1993, 2843.

20. Alkema, H. J.; Arens, J. F. Rec. Trav. Chim. 1960, 79, 1257.

21. (a) Gololobov Yu. G., Soborovskii L. Z. Pat. SU 150502 (1962); Chem. Abstr. 1963, 59, 449g.

(b) Wick, E. L.; Yamanishi, T.; Wertheimer, L. C.; Hoff, J. E.; Proctor, B. E.; Goldblith, S. A. J. Agric. Food. Chem. 1961, 9, 289.

22. Birkofer, L.; Storch, I. Chem. Ber. 1954, 87, 571.

23. Doss, R.C. USA. Patent. 3,419,617, 1968; Chem. Abstr. 1969, 70, 67615.

24. (a) Hon, Y.-S.; Lee, C.-F. Tetrahedron. 2001, 57, 6181. (b) Auge, Y.; Gil, R. Tetrahedron Lett. 2002, 43, 7919. (c) Yu, X.-Y; Liu, C.-H.; Yang, J.-G.; He, M.-Y. Chinese J. Chem. 2006, 24, 1066. (d) Gui, J.; Liu, D.; Chen, X.; Zhang, X.; Song, L.; Sun, Z. Reaction Kinetics Catalysis Lett. 2007, 90(1), 5. (e) Zhu, Z.; Espenson, J. H. Synthesis 1998, 417. (f) Jungnickel, J. L.; Reilly, C. A. J. Molecular Spectroscopy 1965, 16, 135.

25. Ishikawa, M.; Kagawa, N.; Hagiwara, M.; Koboshi, S. Japan Pat. 04024 634, 1992; Chem. Abstr. 1992, 116, 265477.

26. Yamamoto, S.; Usui, A.; Kondo, M. Japan Pat. 04065 412. 1992; Chem. Abstr. 1992, 117, 8760.

27. Segawa, T.; Hosokawa, H.; Yokoro, K.; Uchino, H.; Okada, K. Oyo Yakuri. 1977, 14, 391.

28. Ueno, Y.; Saeki, Y.; Akiyama, T.; Fujita, M. USA Patent 4123525. 1978; Chem. Abstr. 1979, 90, 67760 .

29. (a) Wakasugi, T.; Tonouchi, N.; Miyakawa, T. Chem. Lett. 1992, 171. (b) Harada, Y.; Tanaka, K.; Masuda, H.; Izuimi, K. Japan Patent 08225565. 1996; Chem. Abstr. 1996, 125, 301026.

30. Elamparuthi, E.; Ramaesh, E.; Raghunathan, R. Synthetic Commun. 2005, 35, 2801.

31. Dintzner, M. R.; Matthew, R.; Mondjinou, Y. A.; Pileggi, D. J. Tetrahedron Lett. 2010, 51, 826.

32. Corrochano, P.; Garcia-Rio, L.; Poblete, F. J.; Rodriguez-Dafonte, P. Tetrahedron Lett. 2010, 51, 1761.

33. Zenkevich, I. G.; Ishenko, E. V.; Macarov, V. G.; Macarova, M. N.; Selezneva, A. G. Rus. J. Gener. Chem. 2008, 78, 1682; Chem. Abstr. 2009, 151, 3163.

34. Sasaki, T.; Hayakawa, K.; Nishida, S. Tetrahedron Lett. 1980, $21,3903$. 